Erschienen in: Lang,Ewald/Zifonun, Gisela (Hrsg.): Deutsch - typologisch.

Berlin, New York: de Gruyter, 1996. S. 585-614.

(Jahrbuch des Instituts für deutsche Sprache 1995)

\title{
KAI ALTER
}

\section{Der Zusammenhang von Akzentuierung und Phrasierung im Sprachvergleich*}

\begin{abstract}
Im allgemeinen ist man sich darüber einig, daß ein Zusammenhang zwischen Informationsstrukturierung (Fokus-Hintergrund-Gliederung, Topik-KommentarGliederung) sowie Akzentuierung und prosodischer Phrasierung besteht (vgl. Hayes/Lahiri 1991). Gut untersucht ist die Beziehung zwischen der Unterteilung von Sätzen in prosodische Phrasen und der Fokusstruktur (vgl. Nespor/Vogel 1986). Dies trift ebenso auf die Analyse der Akzentpositionen in Verbindung mit der Informationsstruktur zu (Féry 1993). Bezüglich der Annahmen zur prosodischen Phrasierung und ihrem Zusammenhang mit der Akzentplazierung läßt sich ein solcher minimaler Konsens nur schwer feststellen. Übereinstimmung besteht lediglich darin, daß durch Grenzsignale (Pausen, Glottisverschlufinsertion, Grenztöne) die prosodische Phrasierung manifestiert wird.

Ich möchte hier zeigen, daß zwischen der Akzentplazierung und der prosodischen Phrasierung ebenfalls ein Zusammenhang besteht, und Möglichkeiten erörtern, diese Verbindung sprachübergreifend und experimentell nachzuweisen.

Ich beziehe mich dabei auf Beobachtungen zur Akzentplazierung im Deutschen und Französischen und schlage darauf aufbauend eine Methode zur Determinierung prosodischer Grenzen im Russischen vor.
\end{abstract}

\section{Problemstellung}

Hier soll gezeigt werden, wie mit unterschiedlichen Untersuchungsmethoden der Zusammenhang zwischen Akzentuierung und prosodischer Phrasierung in verschiedenen Sprachen exemplifiziert werden kann. Intonatorische Phänomene wie Akzentplazierung und prosodische Phrasierung stehen in enger Beziehung zu informationsstrukturellen und phonologischen Eigenschaften auf der Satz- und Phrasenebene, ohne daß es immer eine eindeutige Korrelation zwischen linguistischen Kategorien und akustischen Signalparametern gibt. Das liegt am generellen Unterschied von abstrakten phonologischen Beschreibungseinheiten und konkreten signalphonetischen Daten.

- Ich danke Gerhard Jäger, Peter Janker, Ursula Kleinhenz und Ewald Lang für ihre kritischen Bemerkungen und Hinweise. 
Ich gehe davon aus, daß die akustische Realisierung von Akzenten über verschiedene Parameter wie z.B. Grundfrequenzverlauf, Intensität und Länge gesteuert werden kann. Aus dem Bündel der akustischen Parameter wird die Grundfrequenz ausgewählt und anhand des Grundfrequenzverlaufes nach Indizien für die prosodische Phrasierung gesucht.

Die Basis dieser Untersuchung ist in der unterschiedlichen Funktion von Akzenten angelegt: in Sprachen wie dem Französischen können Akzente auch eine demarkative Funktion übernehmen, in anderen Sprachen wie dem Deutschen übernehmen Akzente diese Funktion nur unter bestimmten Bedingungen. Diese Bedingungen werden durch bestimmte Faktoren determiniert, die sich aus der Informationsstrukturierung ergeben.

Im folgenden werden methodische Verfahrensweisen dargelegt, die eine effiziente Bearbeitung akustischer Parameter unter dem Blickwinkel funktional-phonologischer Überlegungen gestatten.

\subsection{Methodologische Vorüberlegungen}

Der beim Sprechvorgang an den Lippen abgestrahlte Schall ist das Resultat komplexer biomechanischer Artikulationsprozesse. Diese Prozesse werden ihrerseits durch neuronale Prozesse gesteuert. Ich beschränke mich hier auf diejenigen meBbaren Vorgänge in dem eigentlich nicht unterbrochenen physikalischen Bereich des signalphonetischen Bandes (Tillmann 1980), der den mittels Mikrophon aufgenommenen und im Oszillogramm sichtbaren Teil des phonetischen Signals betrifft. Damit kann eine Interpretation meBtechnisch ermittelter Daten gewährleistet werden, und, wenn auch in beschränktem Maße, eine Korrelierung dieser Daten mit linguistisch motivierten Beschreibungseinheiten erfolgen.

Zentrales Meßobjekt ist der zeitliche Verlauf des akustischen Signals unter Einbeziehung des Grundfrequenzverlaufes (Fo-Verlauf). Der Grundfrequenzverlauf im akustischen Signal reflektiert die Stimmlippenschwingungen pro Sekunde und hat auf der perzeptiven Seite sein Korrelat im Tonhöhen- bzw. Melodieverlauf (vgl. Abb. 2).

Es wird gezeigt, daß die Einteilung von Äußerungen in kleinere prosodische Gruppen nur bedingt über den Grundfrequenzparameter gesteuert wird, daB auch andere Möglichkeiten existieren, Phrasengrenzen im akustischen Signal zu ermitteln.

Die Grundannahme besteht demnach darin, daß basierend auf der Überlegung von Kleinhenz (in diesem Band) Phrasengrenzen in verschiedenen Sprachen unterschiedlich realisiert werden und ihre Existenz an funktionale Eigenschaften gebunden ist. 
Weiterhin möchte ich annehmen, daß diese Phrasengrenzen akustisch unterschiedlich signalisiert werden und ihre Realisierungsform von den Eigenschaften der lexikalischen Wortakzentuierung abhängt.

\subsection{Untersuchungsmaterial}

Zunächst werden Beispielsätze aus dem Deutschen untersucht und die Schwierigkeiten gezeigt, die bei der Detektion von Phrasengrenzen mittels Fo-Verlauf auftreten. Die untersuchten Sequenzen sind dem von Jacobs (1994) analysierten Material entnommen. Weiterhin wird dafür argumentiert, daB es methodisch angebracht ist, Untersuchungen nicht nur innerhalb einer Einzelsprache vorzunehmen, sondern ähnlich gelagerte $\mathrm{Phänomene}$ auch in anderen Sprachen zu analysieren und aus dem Vergleich des möglicherweise unterschiedlichen Verhaltens der sprachtypischen Parameter AufschluB über das generelle Verhalten der Grundfrequenz bei Phrasierung zu erlangen.

Daher wird auch Material aus dem Französischen und Russischen vorgestellt und diskutiert. Alle drei Sprachen verhalten sich bereits bezüglich der Festlegung des lexikalischen bzw. Wortakzentes sehr verschieden: Russisch ist äußerst variabel bei der Positionierung des Wortakzentes (vgl. zavxóz „Verwalter” und závtrak „Frühstück"), die WortakzentPositionierung im Deutschen unterliegt starken morphologischen und phonologischen Restriktionen, während im Französischen der lexikalische Akzent an der rechten Peripherie von Syntagmen auftritt (vgl. beaucoúp „viel”, mamán „Mama”).

In diesen drei Sprachen wird Prominenz als phonologische Funktion von Wortakzentuierung in unterschiedlichen Positionen realisiert.

Untersucht man Sätze bezüglich ihrer Akzentuierungsvariationen, hat man neben der lexikalischen Ebene für Wortakzentzuweisung die Ebene der Informationsstrukturierung zu berücksichtigen. Die informationsstrukturelle Gliederung eines Satzes wird im Diskurs festgelegt und beeinfluBt u.a. erheblich die Ausprägung der Intonationskontur. Es gibt die Möglichkeit, die Diskursvariabiltät relativ stabil zu halten, und, sieht man von der Untersuchung spontan gesprochener Sprache zunächst ab, durch Fokus-Hintergrund-Gliederung bedingte Effekte differenziert herauszustellen.

Die Frage-Antwort-Methode erlaubt es, die Informationsstruktur eines Satzes zu kontrollieren und Unterschiede in der Informationsgliederung zu analysieren. Durch entsprechende Frageformulierungen können unterschiedliche Fokusstrukturen evoziert werden. 
Hier möchte ich mich auf informationstrukturell unterschiedlich geartete Deklarativsätze beschränken, die als Antwortsequenzen auf Fragen produziert wurden. Die Fragen wurden bezüglich des zu erwartenden Fokustyps unterschiedlich angelegt.

Der Untersuchungsgegenstand ist somit der Grundfrequenzverlauf und die daran ablesbare prosodische Phrasierung von in ihrer Fokusstruktur differierenden Deklarativen im Deutschen, Französischen und Russischen.

Im folgenden möchte ich die theoretische Basis der hier angelegten Untersuchung spezifizieren und Möglichkeiten für die Berechnung der Fokusakzentpositionen darstellen.

\subsection{Theoretischer Rahmen}

Derzeit gibt es verschiedene Möglichkeiten, Akzentuierung und prosodische Phrasierung mit Eigenschaften der syntaktischen und informationsstrukturellen Strukturierung von Sätzen zu verbinden.

Algorithmen an der Schnittstelle zur Phonologie, die sich mit der Ableitung der Position von Satz- und Fokusakzenten befassen, sind bevorzugt im Rahmen der Metrischen Phonologie zu suchen. Sie beziehen sich auf den Übergang einer hierarchisch organisierten syntaktischen Struktur in eine metrische Baum- und/oder Gitterstruktur.

Die strukturelle Position von neutralem Satz- bzw. Fokusakzent ist die der syntaktisch am tiefsten eingebetteten Komponente (Cinque 1993; Abraham 1994; Alter 1994).

Ein ähnlich gelagerter Ansatz, der neben syntaktischen zugleich semantische Informationen berücksichtigt und ebenfalls die Position von Fokusakzenten voraussagt, wird von Jacobs (1994) vorgestellt. Dieses Konzept basiert im wesentlichen auf dem Phänomen der Integration, welches die syntaktischen und informationsstrukturellen Beziehungen $z$ wischen den einzelnen Schwester-Konstituenten im Satz regelt. So ist beispielsweise ein direktes Objekt in einem Satz mit finaler Verbstellung in das Verb integriert, wenn neben anderen semantischen Faktoren der Kontext ein neutraler ist bzw. Normalfokus vorliegt. Aus der Position des Fokusmerkmals läßt sich dann die Satzakzentposition über dem direkten Objekt berechnen:

Was ist passiert?

(1a) Hans hat einem Freund ein HAUS gebaut. 
Ausschlaggebend für die Determinierung der Satzakzentposition ist die Anzahl der errechneten Einträge $\left({ }_{n}{ }^{* n}\right)$. Die Gesamtheit der Einträge bildet ein sog. metrisches Gitter. Die Einträge werden anhand der Spezifizierung der einzelnen Konstituenten für metrische Stärke und Fokus zugewiesen. Sind alle Konstituenten im Satz verarbeitet worden, erhält man eine unterschiedliche Staffelung der Einträge. Diejenige Konstituente, die die meisten Einträge bekommt, ist die prominenteste im Satz:

(1b).... [ einem Freund] [[ein Haus] gebaut $]_{F}$

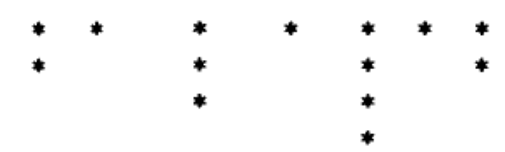

Ohne jetzt im einzelnen auf den exakten Algorithmus für die Ableitung metrischer Bäume und Gitter dieses Satzes bei unterschiedlicher Fokussierung einzugehen, soll hier auf das Phänomen der Fokusambiguität aufmerksam gemacht werden. Die gleiche Sequenz ohne Veränderung der Wortstellung ist auch in einem minimal fokussierten Kontext realisierbar, nämlich auf die Frage:

Was hat Hans einem Freund gebaut?

(2a) Hans hat einem Freund ein HAUS gebaut.

(2b).... [ einem Freund] [[ein Haus $]_{F}$ gebaut]

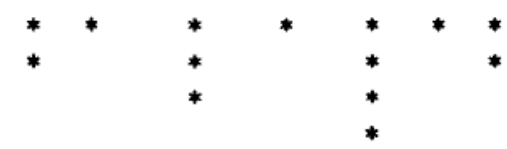

Bei der Ableitung der Prominenzverhältnisse in dieser Antwortsequenz stellt sich heraus, daß der Satzakzent ebenfalls auf dem direkten Objekt liegt und das metrische Gitter in (2b) dem in (1b) gleicht. Ein Unterschied besteht in der Position des Fokusmerkmals, welches in (2) nur das direkte Objekt umfaßt.

Umgekehrt ist eine Disambiguierung anhand der Prominenzverhältnisse dann gegeben, wenn eine andere Konstituente als direktes Objekt eng/minimal fokussiert wird:

Wem hat Hans ein Haus gebaut?

(3a) Hans hat einem FREUND ein Haus gebaut. 
(3b).... [ einem Freund] $\mathbf{F}$ [[ein Haus] gebaut]

Bei dem durch die Frage vorgegeben Kontext rückt alles außer dem indirekten Objekt in den bekannten Hintergrund, und das Fokusmerkmal wird nur dem indirekten Objekt zugewiesen. Daraus wird dann die Position des prominentesten Akzentes berechnet.

Ein ähnlicher Effekt tritt bei multipler Fokussierung, ausgelöst durch multiple w-Fragen, auf: Sowohl dem indirekten als auch dem direkten Objekt wird ein separates Fokusmerkmal zugewiesen, und beide erhalten gegenüber den anderen Konstituenten entsprechend mehr Einträge im metrischen Gitter, wobei zu beachten ist, daB das direkte Objekt, ausgezeichnet als das designierte terminale Element, einen Eintrag zusätzlich im Vergleich zu seiner linksadjazenten Schwester bekommt.

Was hat Hans wem gebaut?

(4a) Hans hat einem FREUND ein HAUS gebaut.

(4b).... [ einem Freund $]_{F}\left[[\text { ein Haus }]_{F}\right.$ gebaut]

Dieser Ansatz gestattet es, eine syntaktische Struktur derart zu verarbeiten, da $B$ die Position des Satzakzentes voraussagbar wird. Diese Position ist diejenige, die die meisten Einträge in einem metrischen Gitter erhält.

Wird das direkte Objekt eng fokussiert oder kontrastiert, ergibt sich die gleiche metrische Gitterkonstellation wie bei neutraler Fokussierung. Der Satzakzent fällt wegen Integration und/oder syntaktischer Einbettungstiefe im deutschen Verb-letzt-Satz bei neutraler Fokussierung auf das direkte Objekt. Das Fokusmerkmal projiziert in diesen Fällen über einen maximalen Bereich, der nur durch Topikalisierung unterbrochen wird, während in anderen Kontexten die Projektion von Fokus eingeschränkt wird und sich u.U. nur auf das direkte Objekt bezieht. Die sog. Fokusprojektion kann aber durch Fokussierung anderer Konstituen- 
ten aufgebrochen werden, und genau das beeinflußt auch die prosodische Phrasierung.

Jedoch sind die Voraussagen metrischer Transformationen bezüglich der prosodischen Phrasierung restringiert, da sie ausschließlich an die Position der metrisch stärksten Einheit gebunden sind.

Ein Modell, welches sich in der Nähe der Schnittstelle zur Phonetik/Akustik ansiedelt, ist das Tonsequenzmodell (vgl. Pierrehumbert 1980). Dieses Modell inkorporiert gleichsam eine metrische Komponente und transformiert die relevanten Informationen in eine tonale Komponente.

\subsubsection{Der Ton-Sequenz-Ansatz}

Dieser theoretische Ansatz erlaubt neben der Ableitung der Prominenzverhältnisse mittels Einträgen der metrischen Gitter zusätzlich eine Annäherung an den Grundfrequenzverlauf in einem Satz. Der Fo-Verlauf wird in einer tonalen Komponente aus den Informationen der metrischen Repräsentationsebene und der Spezifizierung des Fokustyps gewonnen und über Interpolationsregeln in eine akustische Form übergeleitet.

Damit ergibt sich die Möglichkeit, extrahierte Grundfrequenzverläufe zu interpretieren. Fo-Verläufe können reanalysiert werden, indem zwei interagierende Tonniveaus angenommen werden: Hoch- und Tieftöne. Die aus den Tönen kombinierten Tonsequenzen bilden eine zugrunde liegende Form im Sinne einer prosodischen Realisierung von Konturen bzw. Melodieverläufen und dienen als Eingabe für die Ableitung des Grundfrequenzverlaufes der Äußerung.

Eine Äußerung ist in kleinere prosodische Einheiten - in Intonationsphrasen und intermediäre Phrasen - zerlegbar. Jede Phrase enthält eine Sequenz von Hoch- und Tieftönen, die wiederum in 3 Typen klassifizierbar sind: Akzenttöne, Phrasentöne und Grenztöne. Akzenttöne können mono- oder bitonal auftreten. Sie werden über zentrale Assoziierung den minimalen tontragenden Einheiten zugewiesen. Über Merkmalsperkolation werden die als metrisch stark ausgewiesenen Silben mit Akzenttönen assoziiert.

Grenztöne werden der letzten Silbe der Phrase zugewiesen, Phrasentöne stehen zwischen Akzent- und Grenztönen. Die Zuweisung von Grenztönen an Phrasengrenzen erfolgt über periphere Assoziierung. Die folgende Graphik veranschaulicht den Aufbau des Tonsequenzmodells in Auszügen. Wie auch in der Autosegmentalen Phonologie wird von einem multidimensionalen Aufbau der einzelnen Ebenen ausgegangen. In 
Abb. (1) sind die prosodische, die tonale und die phonemische Ebene dargestellt. Die prosodische Ebene enthält die relevanten Informationen zur prosodischen Hierarchie und damit zum Aufbau der prosodischen Phrasen und der darunter liegenden Ebenen. Die tonale Ebene kodiert die Informationen über die Zuweisungsdomäne von Akzenttönen und Grenztönen. Im vorliegenden Fall werden Akzenttöne der Domäne "pitch accent" (PA) zugewiesen und Grenztöne mit den Grenzen intermediärer (ip) und Intonationsphrasen (IP) assoziiert. Die phonemische Ebene erhält zugleich Informationen aus der prosodischen Ebene, z.B. über den Aufbau der Silbenstruktur, und setzt die Informationen über die Tonstruktur der tonalen Ebenen um.

Dieses Informationspaket, insbesondere jedoch die Angaben über die tonale Struktur, kann dann mittels Interpolationsregeln in einen Grundfrequenzverlauf transformiert werden.

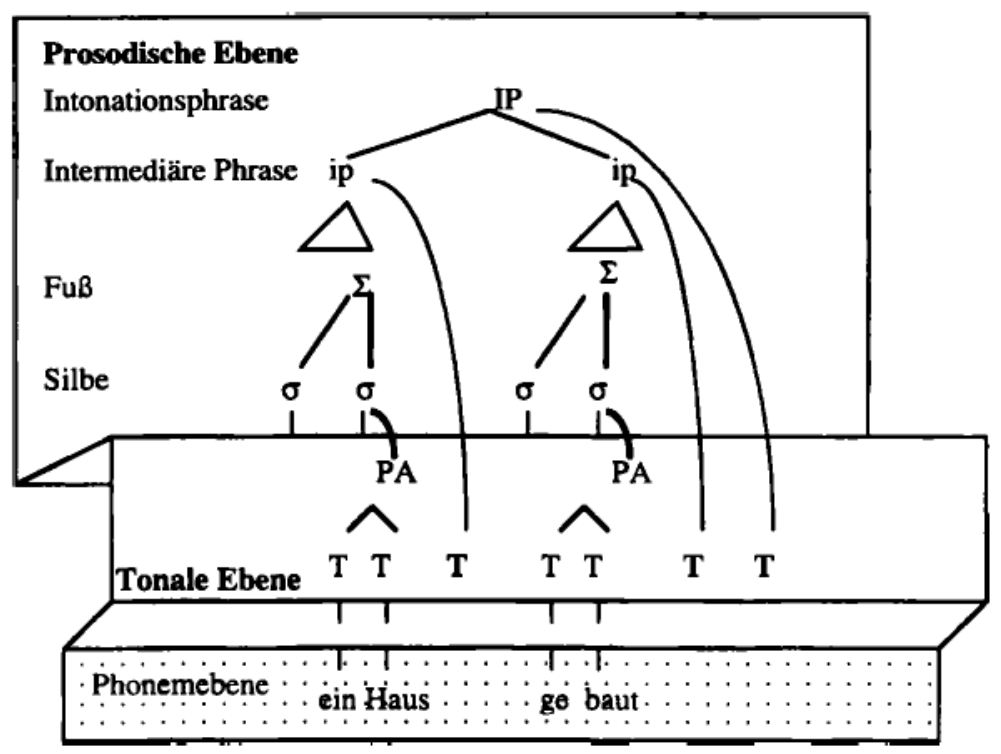

Abb.1: Der schematisierte Aufbau einzelner Ebenen im TonsequenzModell für den Abschnitt „...ein Haus gebaut”.

Nicht unumstritten ist allerdings die Zuweisungsdomäne für die verschiedenen Typen von Tönen. Erschwerend tritt hinzu, daß Akzenttöne und Grenztöne direkte funktionale Korrelate (Fokus resp. prosodische Phra- 
sierung) haben, diese aber akustisch nicht immer exakt determinierbar sind. Der Bezug zum Fo-Parameter genügt daher nicht immer, um alle Facetten einer globalen Kontur und diskursrelevante Varietäten zu erfassen. Anzeichen dafür gibt es aus aktuellen Untersuchungen von Dogil (1995) für das Deutsche, wo der Wortakzent (i.e. Akzent in neutraler Position) auch über den Zeitparameter und der Fokusakzent über den Grundfrequenzverlauf gesteuert werden kann. Selbiges gilt auch für die Detektion prosodischer Phrasengrenzen (s. Batliner 1994).

Damit ist der theoretische Rahmen für die folgenden experimentellen Untersuchungen vorgegeben: Die Informationsstruktur wird in eine syntaktische Struktur mittels Merkmalen implementiert. Mit diesen strukturellen Vorgaben lassen sich in einer metrischen Komponente die Prominenzverhältnisse im Satz berechnen. Eine tonale Komponente gibt zusätzlich dazu Informationen über die prosodische Phrasierung und die tonale Struktur, womit eine maximale Annäherung an die tatsächlich realisierte akustische Form erreicht werden kann. Auf diesem Hintergrund lassen sich nun die folgenden Arbeitshypothesen aufstellen, die experimentell verifiziert werden sollen.

\subsection{Arbeitshypothesen}

Es gibt, wie oben diskutiert, einen Zusammenhang zwischen Akzentplazierung, Akzenttyp und Informationsstrukturierung. Dieser Zusammenhang soll um die Kategorie "Phrasierung” erweitert und am Verhalten des Grundfrequenzparameters nachgewiesen werden. Dabei wird gezeigt, inwieweit dieser Parameter für den Nachweis informationsstruktureller Eigenschaften strapazierbar ist.

Die Grundfrequenzbewegung (Fo) hat (neben anderen Funktionen) die Aufgabe

a) die Position der Fokusakzente anzuzeigen,

b) die Art des Fokus zu signalisieren,

c) die Grenzen von prosodischen Einheiten zu markieren.

\subsubsection{Beobachtungen im Deutschen}

Diejenigen Silben, die Träger für lexikalischen Wortakzent sind, tragen auch den Fokusakzent. Das sagt allerdings weder etwas über die $\mathrm{Zu}$ weisungsdomäne für Fokusakzente aus, noch sind Voraussagen über die Phrasierung möglich.

Die oben genannten Schwierigkeiten für die Zuweisung von Akzentund Grenztönen und deren akustische Realisierung sollen im folgenden an ausgewählten Beispielen des Deutschen exemplifiziert werden. Dazu 
wurde ein Teststimulus nach der Frage-Antwort-Methode aufgebaut und die von Jacobs (1994) untersuchten Sätze wurden aufgezeichnet, digitalisiert und einer meBtechnischen Analyse unterzogen. Dies betriftt die folgenden Frage-Antwort-Paare:

(5) Was ist passiert?

Hans hat seinem Freund ein HAUS gebaut.

$$
\text { (=Normalfokus) }
$$

(6) Was hat Hans seinem Freund gebaut? (=Fokus auf dem) Hans hat seinem Freund ein HAUS gebaut. direkten Objekt)

(7) Hans hat wem was gebaut? Hans hat seinem FREUND ein HAUS gebaut.

(=multiple Foki)

Anhand von Fo-Verläufen und Oszillogrammen soll nun nach der Fokusakzentposition, der durch den Fo-Verlauf induzierten Fokusart und der durch Fokussierung bedingten Phrasierung gesucht werden.

Die graphischen Darstellungen in Abb. (2) sind folgendermaBen zu interpretieren: Im oberen Teil des Gesamtdiagramms ist der oszillographische Verlauf, d.h. die Schalldruck-Zeit-Funktion als Resultat der Membranschwingung des Mikrophons abzulesen, im unteren Teil ist der aus dem Signal extrahierte Fo-Verlauf zu sehen. Auf der x-Achse ist der zeitliche Verlauf der Grundfrequenz in Millisekunden ablesbar, die y-Achse zeigt die Werte der Grundfrequenz in Hertz an. Das Gesamtdiagramm beinhaltet die digitalisierte und damit computerlesbare Information jeweils einer Antwortsequenz.

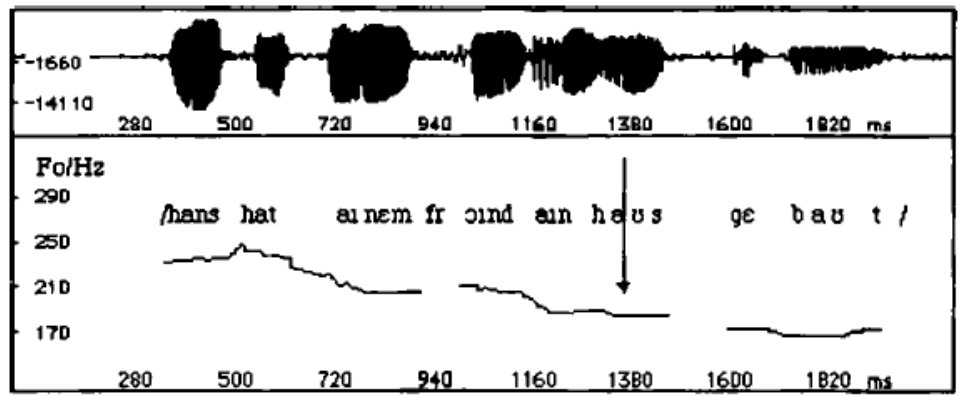

Abb. 2: Oszillogramm und Fo-Verlauf von Beispiel (5), die mit Pfeil markierte Stelle ist die Position des Fokusakzentes bei Normalfokussierung. 
Bevor die Position und die Form der einzelnen Akzente interpretiert werden kann, ist einem bio-mechanisch basierten ProzeB Rechnung zu tragen - dem der Deklination. Dieser ProzeB bewirkt das stetige Absinken der Grundfrequenz zum Satzende hin. Dies ist am gleichmäßig fallenden globalen Kurvenverlauf in Abb. (2) erkennbar.

Da Fokusakzente in dem hier untersuchten Material auf den Bereich des direkten und indirekten Objekts beschränkt sind, werden auch nur diese Sequenzen näher betrachtet.

Zunächst fallt auf, daB die Fokusposition anhand des Fo-Verlaufes nicht einfach zu bestimmen ist. Aufgrund der Voraussagen aus der Metrik kann davon ausgegangen werden, da $B$ das direkte Objekt ein Haus Träger der Fokus- resp. Satzakzentposition ist. Der Fokustyp "Normalfokus" beeinflußt den tonalen Verlauf über dieser Position. Dieser Verlauf kann als, wenn auch schwach ausgeprägtes, Fallen beschrieben werden.

Eine Phrasierung sollte nach den Regeln der Fokusprojektion zwischen indirektem und direktem Objekt nicht existieren, da in diesem Satz ein maximaler Bereich fokussiert ist.

In Abb. (3) ist der Fo-Verlauf aus Beispielsatz (6) zu sehen. Hier wird das direkte Objekt minimal fokussiert, es ist auch Träger des Satzakzents (s.o.). Der Tonhöhenverlauf zeigt im Gegensatz zu (5) keine signifikanten Unterschiede. In diesem Beispielsatz gibt es ebenfalls keine Indizien für eine Phrasengrenze zwischen indirektem und direktem Objekt.

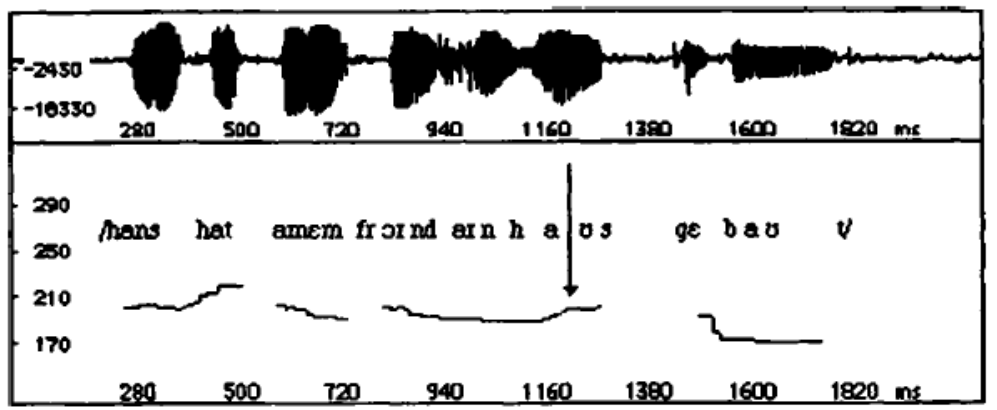

Abb. 3: Oszillogramm und Fo-Verlauf von Beispiel (6), die mit Pfeil markierte Stelle ist die Position des Fokusakzentes bei enger Fokussierung des direkten Objekts. 
Im Vergleich dazu ist der Fall der multiplen Fokussierung in Abb. (4) bezüglich der Akzenttypen und auch der Phrasierung eindeutig interpretierbar. Deutlich sind die zwei Fokusakzente als Fo-Anstieg über beiden Objekten realisiert. Damit ergibt sich auch die Möglichkeit, anhand des Fo-Verlaufs eine Phrasengrenze zu interpretieren.

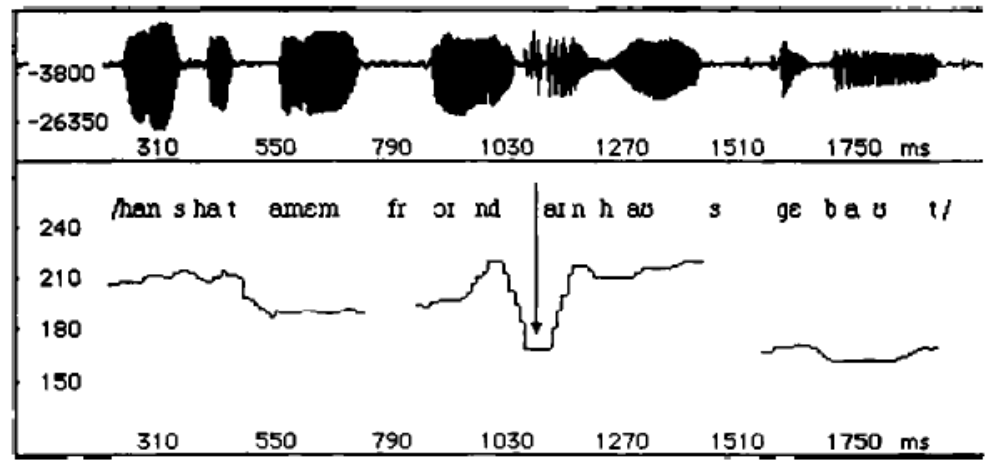

Abb. 4: Oszillogramm und Fo-Verlauf von Beispiel (7), die mit Pfeil mar kierte Stelle ist die Position der Grenze zwischen den beiden eng fokussierten Konstituenten.

\subsubsection{Interpretation der Daten im Tonsequenzansatz}

Die Beispiele zeigen, dab es charakteristische natürliche Tendenzen für die Realisierung unterschiedlicher Fokusstrukturen und der damit verbundenen Phrasierung gibt (vgl. Féry 1993 und Uhmann 1989).

Jede Ebene der prosodischen Hierarchie ist phonologisch motiviert und hat Verbindungen zu anderen Ebenen der Grammatik (Syntax, Informationsstruktur).

Sprachen können sich dadurch unterscheiden, welche der Ebenen die wichtigste für Akzentzuweisung und Grenzmarkierung ist. Dabei können verschiedene Domänen miteinander konkurrieren, genauso wie es möglich ist, daB in einer Sprache bestimmte Ebenen nicht relevant sind. Unter Berücksichtigung informationsstruktureller Gegebenheiten ist es wichtig, diejenige Domäne zu spezifizieren, die stärker an syntaktischinformationsstrukturelle Einheiten gebunden ist, um die prosodische Strukturierung angemessen ableiten zu können. 
Ohne auf die kontroverse Diskussion zum Inventar prosodischer Einheiten einzugehen, will ich zeigen, daß die zwei höchsten prosodischen Domänen relevant für Fokuseffekte sind: im vorgestellten Modell die Intonationsphrase und intermediäre Phrasen.

Jede intermediäre Phrase ist potentielle Trägerin eines separaten Akzenttons. Dies wird deutlich im Fall (7) mit multipler Fokussierung. Die weniger eindeutigen Fälle (5/6) bedürfen einer zusätzlichen Erläuterung.

Neutraler bzw. globaler Fokus induziert einen maximalen Fokusbereich und nur die integrierte Konstituente, das adjazent zum Verb stehende direkte Objekt sticht als prominent hervor.

Dies ist ebenso der Fall bei minimaler Fokussierung des direkten Objekts wie in (6). Vergleicht man beide Fälle, so zeigt sich Fokusambiguität, d.h., beide Sätze sind prosodisch nicht voneinander zu unterscheiden.

Im Rahmen des Tonsequenzansatzes findet sich eine Erklärung für diesen Effekt. Bei normalem Fokus dominiert das Fokusmerkmal eine Intonationsphrase, die indirektes und direktes Objekt umschließt. Bei minimalem Fokus (6) dominiert das Fokusmerkmal die tiefer eingebettete Konstituente des direkten Objekts, allerdings ändert sich an der prosodischen Phrasierung nichts (vgl. Nespor/Vogel 1986). Die eng fokussierte Objektphrase formt innerhalb der sie dominierenden Intonationsphrase eine eigene intermediäre Phrase (ip), die zugleich die Domäne für die Zuweisung des Fokusmerkmals ist.

(8) ... [seinem Freund [ein Haus] $\left.]_{\text {ip }}\right]_{I P}$ gebaut

Das Fokusmerkmal perkoliert auf die höhere Ebene der Intonationsphrase und wiederum werden beide Objekte von diesem Merkmal dominiert. Damit ergibt sich keine zwingende Notwendigkeit, ein Grenzsignal vor dem direkten Objekt einzufügen.

Im Gegensatz dazu steht Beispiel (7) mit multipler Fokussierung, wo jeder Objektphrase ein Fokusmerkmal zugewiesen wird und beide dadurch eigene prosodische Phrasen bilden können.

(9) ... [[seinem Freund $]_{i p}$ [ein Haus $\left.]_{i p}\right]_{I P}$ gebaut

Damit ergibt sich, daB jede fokussierte Konstituente eine eigene prosodische Phrase auf den unterschiedlichen Ebenen bilden kann und dann auch, wie im folgenden demonstriert, eine Domäne für die Fokusakzentzuweisung bietet.

Gussenhoven (1994) argumentiert für eine radikale Annahme der prosodischen Struktur für das Englische: Es gibt bei normalem Fokus, keine 
Indizien für Phrasierung bzw. Grenztonrealisierung sondern nur bei multiplem Fokus.

Zunächst wird jeder Konstituente ein bitonaler Akzentton der Form $\mathrm{H}^{*} \mathrm{~L}$ zugewiesen. ${ }^{1}$ Dieser Akzentton bedeutet, daB über der akzentuierten Silbe ein von einem hohen Niveau (H für high) auf ein niedrigeres Niveau (L für low) fallender tonaler Verlauf erzeugt wird.

Bei weiter Fokussierung wandert der zweite Ton (L) des präfinalen bitonalen Akzentes nach rechts und endet an der linken Seite des folgenden Akzentes (vgl. unten für das Deutsche). Die Verbindung zwischen beiden Akzenten wird durch Interpolationsregeln hergestellt. Bei Normalfokus gibt es prosodische Restrukturierung, bei multiplen Foki wird die ursprüngliche, d.h. die syntaktische Phrasierung weitestgehend beibehalten.
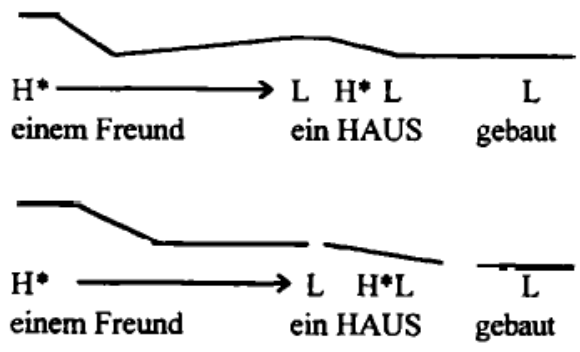

Man kann hier annehmen, daB im Fall (10) $(=(5))$ das Fokusmerkmal oberhalb der Domäne der intermediären Phrasen operiert und die gesamte Verbhülle dominiert. Die Tonverschiebung ist daher möglich, weil keine Eigenschaft die Insertion von Grenzmarkern zwingend erfordert. Ähnliches gilt auch im Beispiel (11) (=(6)). Das Fokusmerkmal dominiert zunächst die intermediäre Phrase, bestehend aus direktem Objekt, perkoliert dann aber an die Intonationsphrase, womit die Fokusdomäne erweitert wird. Dadurch werden Grenzmarker vermieden, der Akzentton erfährt die gleiche Realisierung wie in (5), woraus Fokusambiguität resultiert.

1 Es wird im allgemeinen davon ausgegangen, daß Fokusakzente im Englischen und Deutschen bitonal sind. 


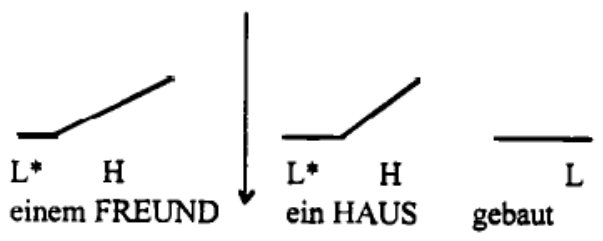

In (12) dominiert je ein Fokusmerkmal eine syntaktische Konstituente, beide Merkmale bewirken die Bildung zweier prosodischer Phrasen, über denen je ein bitonaler Akzentton realisiert wird. Die Tonhöhenbewegung weicht von der in den vorangegangenen Beispielen $a b$, da in (12) ein andere Fokustyp vorhanden ist und daher vom Sprecher eine andere Tonsequenz aus dem Toninventar ausgewählt wird.

\subsubsection{Zusammenfassung}

Die Hypothesen (a) und (b) aus Abschnitt 1.4 oben konnten anhand der akustischen Untersuchung der hier ausgewählten Beispiele bestätigt werden: Die Tonhöhenbewegung zeigt zugleich die Position und die Art der Fokusakzente an.

Weniger eindeutig ist die prosodische Phrasierung am Grundfrequenzverlauf ablesbar. Das hängt mit der Eigenschaft des Deutschen zusammen, Wortakzente nicht demarkativ zu verwenden, sondern ihnen eine lexikalische Funktion zuzuweisen.

Bildet man jedoch Extremfäle, wie bei multipler Fokussierung, und vergleicht zwei eng fokussierte, adjazent zueinander stehende, einsilbige Konstituenten miteinander, läßt sich am Tonhöhenverlauf die prosodische Strukturierung erkennen. Dies soll jedoch nicht ausschließen, daB es andere Möglichkeiten für die Signalisierung von prosodischen Grenzen gibt, z.B. die Glottisverschlußinsertion und Assimilationserscheinungen. Diese wurden im Rahmen der vorliegenden Untersuchungen nicht analysiert.

Bezüglich der akustischen Realisierung der mittels metrischer Algorithmen errechneten Prominenzverhältnisse in Sätzen muß die Untersuchung erweitert werden, was hier nicht geleistet werden soll. Der Verweis darauf, daß Prominenz ein ganzes Merkmalsbündel akustischer Parameter 
umfaBt (z.B. Umfang der Tonhöhenbewegung, Dauerverhältnisse, Intensität usw.) und sie im Deutschen nicht direkt am Fo-Verhalten über den relevanten Positionen erkennbar ist, soll hier genügen.

Im folgenden wird eine Sprache untersucht, die Akzente in fester Position hat und diesen eine demarkative Funktion zuweist - das Französische.

\section{Beobachtungen im Französischen}

Die aktuelle Diskussion zur Phonologie des Französischen betrifft den Zusammenhang zwischen Silbifizierungsregeln und Akzentuierung (vgl. Tranel 1987 und 1995), insbesondere die oft beschriebenen Phänomene der Liaison, Verkettung (enchainement) und Schwa-Tilgung.

Im weiteren möchte ich die eben genannten phonologischen Prozesse im Zusammenhang mit Akzentuierungsregeln exemplifizieren. Es zeigt sich, daß einige Silbifizierungseffekte nur bedingt unabhängig von Einflüssen der Akzentuierung im Französischen auftreten.

Erst dann möchte ich eine an die vorherige Analyse des Deutschen anschließende Beschreibung der Akzentuierungsphänomene im Zusammenhang mit Fokussierungsstrategien im Französischen geben.

\subsection{Die Liaison}

Zusätzlich zu den in Nespor/Vogel (1986; vgl. Kleinhenz in diesem Band) beschriebenen Daten bezüglich der durch die Phrasierung blockierten Liaison möchte ich weitere Daten aus der einschlägigen Literatur (z.B. Lyche/Girard 1995) und eigene Beobachtungen anführen. Liaison, Schwa-Tilgung und Resilbifizierung sind phonologisch eng zusammenhängende Prozesse: Der betroffene Konsonant kann in den Onset einer Silbe eines folgenden Wortes oder aber in die Coda der letzten (betonten) Silbe des vorangehenden Wortes verlagert werden. Diese Prozesse sind nur dann voneinander zu differenzieren, wenn die Position und damit zusammenhängend die Art des Akzentes korrekt berücksichtigt werden. Ich möchte zeigen, daß die Resilbifizierung und die Zuweisung von Fokusakzenten miteinander in Verbindung stehen.

Folgende zwei Typen der Liaison sind in Relation zur Verkettung im Französischen zu unterscheiden: nicht-verkettete (13) und verkettete (14) Liaison.

(13) J'avais ... un rêve

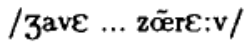
ich hatte einen Traum 
(14) J'avais ... un rêve

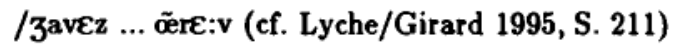
ich hatte einen Traum

Die Liaison vom Typ (13) kann allerdings dann verhindert werden, wenn das folgende Wort initialen Akzent trägt:

(15) Il y a une incroyable pudeur (vgl. Lyche/Girard 1995, S. 216)

/i li ja yn ... Ẽkrwajablə pydœ:r/

*/i li ja y ... n Ẽkrwajabla pydœ:r/

es gibt eine unglaubliche Schamhaftigkeit

Dieses Phänomen ergibt sich aus der Tatsache, daß aufgrund der Betonung der ersten Silbe ein Glottisschlag eingesetzt wird und daher keine Position mehr frei ist für den Konsonanten der Coda des vorangehenden Wortes.

Hier liegt auch der fundamentale Unterschied zwischen beiden Typen der Liaison (13/14). Die nicht-verkettete Liaison ist obligatorisch, die verkettete fakultativ. Sie wird daher in der einschlägigen Beschreibung auch unter der Bezeichnung "fakultative Liaison" geführt.

Die obligatorische Liaison tritt aber im Gegensatz zu (15) auch dann auf, wenn die initiale Silbe des nachfolgenden Wortes betont wird:

(16) Un appui

/थ̃ ... napqi/

eine Unterstützung

Andere Beispiele verweisen darauf, daB es möglicherweise einen weiteren Zusammenhang zwischen der Plazierung des Akzentes, also initial vs. final, und der Liaison gibt.

Son imagination seine Vorstellungskraft

(a) /sñimaวinasjj̄ bei Endbetonung

(b) /sõ...nimazinasjõ/ bei initialer Betonung

Dennoch lassen sich systematische Unterschiede zwischen Akzentplazierung und dem Vorkommen der Liaison verschiedenen Typs feststellen, wenn längere Sequenzen untersucht werden.

Betrachtet man die folgenden Beispiele, läBt sich erkennen, daß bei initialer Betonung von (I) (vgl. 18c) der rechte Wortrand des Wortes (I) nicht von der Liaison betroffen wird, während bei finaler Betonung in (II) sowohl der linke Wortrand von (II) als auch der rechte Wortrand von (I) einer Resilbifizierung durch die Liaison unterzogen werden. 


$$
\begin{aligned}
& \text { I } \\
& \text { un chemin de campagne } \\
& \text { ein Weg des Feldes (= ein Feldweg) }
\end{aligned}
$$

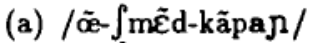

bei Endbetonung auf II

(b) / $\tilde{e}-\int \mathrm{m} \tilde{\varepsilon}$-dkäpan/

bei initialer Betonung auf II

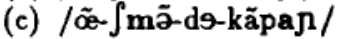

bei initialer Betonung auf I

Im Beispiel (18a) tritt die verkettete Liaison auf, der Konsonant der Präposition de wird in die Coda des vorangehenden Wortes (I) verschoben. In (18b) trifft man auf die nicht-verkettete Liaison, wobei der Konsonant der Präposition de nach der Tilgung des Schwa an den ersten Konsonanten der ersten Silbe des folgenden Wortes verschoben wird, jedoch die Konstituente I intakt bleibt. Bei Betonung des Wortes (I), also bei initialer Betonung der gesamten Kombination, wird weder das Schwa der Präposition getilgt, noch tritt eine Variante der Liaison auf, d.h. die Grenzen des folgenden Wortes bleiben intakt.

Die oben genannten Beispiele (16) und (17) sind, wie ich im folgenden ausführen möchte, nicht als Gegenbeispiele zu (15) oder (18c) zu interpretieren. Dazu schlage ich folgende Interpretation für (16/17) vor: In diesen Beispielen ist die Liaison obligatorisch, in (15) wird sie aber durch die Einsetzung des Glottisverschlusses blockiert. Man kann hier annehmen, da $B$ in (15) durch die initiale Akzentuierung des Adjektivs dieses eine selbständige prosodische Phrase bildet, während in (16/17) trotz initialer Betonung der Nominalphrasen diese neben dem Indefinitartikel und dem Possessivum keine eigenständigen prosodischen Phrasen formen. Die Beispiele (16/17) sind dann lediglich ein Indiz dafür, daß die obligatorische, d.h. die nicht-verkettete, Liaison unabhängig von der Akzentplazierung stattfindet, wenn durch die initiale Akzentuierung kein besonderer Fokustyp ausgedrückt wird. Wären die Nominalphrasen in (16/17) eng fokussiert, würde sich der Akzent keinesfalls verlagern, sondern die Art des Akzentes würde sich verändern.

Die Beispiele unter (18) verweisen jedoch auf ein weiteres Phänomen. Der Vergleich zwischen (18a) und (18b) zeigt, daß bei initialer Betonung der Konstituente II campagne die Konstituente I chemin am rechten Wortrand intakt bleibt und der Onset der betonten Silbe in Konstituente II ein selbst für das Französische ungewöhnliches Konsonantencluster bildet.

Die Beispiele (18b) und und (18c) haben gemeinsam, daß generell bei initialer Betonung rechte Wort- bzw. Phrasengrenzen nicht der Liaison 
unterzogen werden. Die Konstituente I chemin bleibt in beiden Fällen an ihrem rechten Rand im ursprünglichen Zustand.

Daraus läßt sich ableiten, daß drei Prozesse miteinander interagieren die Akzentverlagerung auf die erste Silbe einer Konstituente, die Konservierung des rechten Phrasenrandes dieser Konstituente sowie die der rechten Peripherie der vorangehenden Konstituente.

Im weiteren möchte ich zeigen, daß es externe Faktoren der Informationsstruktur sind, die eine Verlagerung des Akzentes bedingen können und dadurch die eigentliche prosodische Phrasierung aufbrechen. Diese Prozesse sind nicht nur eine Strategie zur Konservierung der Autonomie kleinerer prosodischer Einheiten wie z.B. Wort (vgl. die Argumentation von Lyche/Girard 1995). Sie können auch aus einer Sicht erklärt werden, die sich auf die Kategorie Fokus bezieht und das Erklärungspotential wesentlich erweitert.

Im folgenden möchte ich demonstrieren, daB initiale Akzentuierung von bestimmten Konstituenten dazu dient, Unterschiede in der Fokusstruktur von Sätzen zu signalisieren. Das Untersuchungsobjekt sind hierbei Adverbien, die über spezielle syntaktische Eigenschaften verfügen.

\subsection{Vorüberlegung zum Experiment}

Ähnlich der für das Deutsche dennonstrierten Methode wurden Minimalpaare nach der Frage-Antwort-Methode gebildet, wobei jedoch im Französischen andere Konstituenten erfragt wurden.

Der Test bezieht sich hier auf Adverbien, die in ihrer Positionierung im Satz bestimmten syntaktischen Restriktionen unterliegen. Gradadverbien können im Französischen nicht an die Satzspitze bewegt oder linksdisloziiert werden.

(19) *C'est énormément ce qu'il a fumé.

es ist enorm viel was er geraucht hat

(20) *Enormément a-t-il fumé.

enorm viel hat er geraucht

Anders verhalten sich dagegen Objekte. Sie können an die Satzspitze bewegt werden und signalisieren durch ihre veränderte Position im Satz einen bestimmten Fokustyp.

(21) C'est Wim que Anne aime. es ist Wim den Anne mag

Gradadverbien können jedoch sowohl vor als auch nach dem Partizip stehen: 
(22) Il a énormément fumé.

er hat Adv. geraucht

(23) Il a fumé énormément.

er hat geraucht Adv.

Das von Objekt-Phrasen verschiedene Verhalten der Adverbien bezüglich ihrer Positionierung im Satz soll in der folgenden Untersuchung dahingehend ausgenutzt werden, daB sie bei Verschiebung im Mittelfeld des Satzes die prosodische Phrasierung verändern können. Dies ist bedingt durch ihren kategoriellen Status - sie sind Maximalphrasen (vgl. Hirst 1993). Werden sie zudem eng bzw. kontrastfokussiert, verlagert sich der Akzent auf ihre erste Silbe. Hierbei wäre wie in (15) zu erwarten, daB ein Glottisschlag als Grenzsignal vor der betonten Silbe eingefügt wird. Nur in $60 \%$ der untersuchten Fälle konnte jedoch ein Glottisschlag beobachtet werden (vgl. Tranel 1987, der auf die Optionalität der GlottisverschluBinsertion bei emphatischer Hervorhebung von Adverbien verweist). Damit stellt sich die Frage, wie bei bestimmten Fokusarten eine Veränderung in der prosodischen Phrasierung akustisch determinierbar ist. Im weiteren wird gezeigt, daB der bei der Phrasierung beteiligte relevante Parameter der Fo-Verlauf ist.

\subsubsection{Datenerfassung}

Es tritt der Effekt auf, daB bei kontrastiver Fokussierung des Adverbs die erste Silbe dieser Konstituente betont wird. Im Gegensatz dazu steht der Fall der Normalfokussierung, wobei die lexikalische Betonung beibehalten wird und die letzte Silbe des Adverbes betont bleibt.

Qu'est-ce qui s'est passé?

Was ist passiert? (= Normalfokus)

(24a) Il a fumé énormément.

(24b) Il a énormément fumé.

Unter Normalfokussierung und bei Kontrastfokus sind beide Stellungsvarianten des Adverbes möglich.

(25) A-t-il fumé beaucoup ou peu?

Hat er viel oder wenig geraucht? (= kontrastiver Fokus)

(25a) Il a fumé énormément.

(25b) Il a énormément fumé.

Bei Kontrastfokus wird hingegen die erste Silbe der erfragten Konstituente, hier des Adverbs, betont.

Aus dem Gesamtkorpus möchte ich an zwei ausgewählten Grundfrequenzverläufen belegen, daB tatsächlich Unterschiede in der prosodischen 
Strukturierung zwischen normal- und kontrast-fokussierten Sätzen auftreten.

Die Voraussetzung dafür ist, daB der Grundfrequenzparameter ein mögliches akustisches Korrelat für Akzentuierung im Französischen ist ${ }^{2}$ und da $\mathrm{B}$ aus phonologischer Sicht die Position und weniger die Art des Akzentes verschiedene Fokustypen anzuzeigen vermag.

Die folgende Abbildung zeigt das Oszillogramm und den dazugehörigen Grundfrequenzverlauf eines aufgezeichneten Satzes (24b). Der Kontext, hier die entsprechende Frageformulierung, verweist auf einen normalfokussierten Antwortsatz.

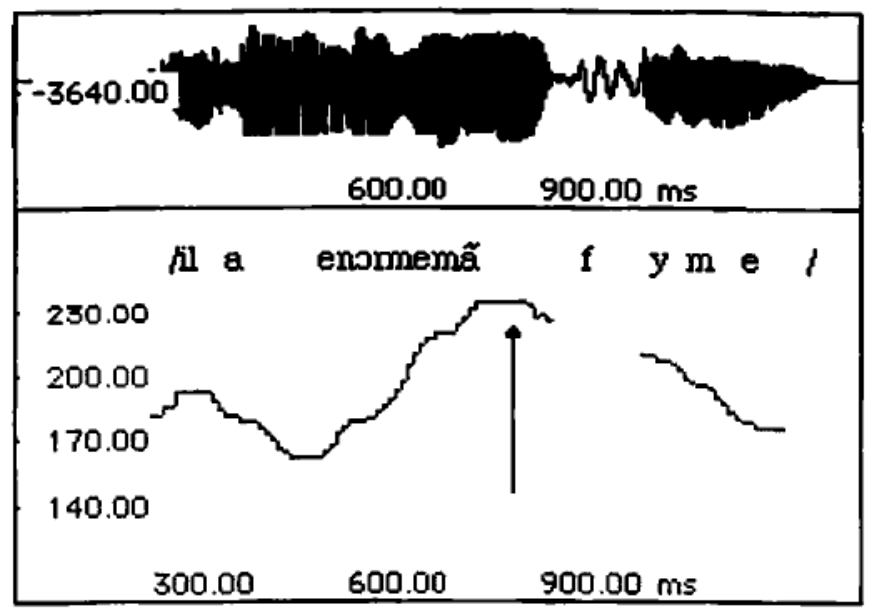

Abb. 5: Der Pfeil markiert die Stelle zwischen der rechtsseitigen Grenze des Adverbes und dem Frikativ, der linksseitigen Begrenzung des Partizips.

Wie der Abbildung zu entnehmen ist, zeigt der Gipfel des Grundfrequenzverlaufes über der letzten Silbe des Adverbs einen Akzent an; der Grundfrequenzfall über der letzten Silbe des Partizips ist durch den Satzmodus bedingt - hier ein Deklarativ, und markiert somit eine weitere Akzentposition und die rechte Satzgrenze.

2 Vgl. dazu Caelen-Haumont (1993) über die wichtigsten akustischen Korrelate von Akzentuierung im Französischen. 
Ein völlig abweichendes Verhalten des Fo-Verlaufs ergibt sich bei Kontrastfokussierung im Beispiel (25b). Obwohl die Wortfolge die gleiche wie in (24b) ist, ergibt sich ein anderes Bild: Anhand der Grundfrequenzbewegung über der ersten Silbe des Adverbs (s. Pfeil) kann davon gesprochen werden, daß sich an dieser Position der Phrasenakzent befindet.

Der finale Fall über dem Partizip verläuft ähnlich wie in Satz (24b).

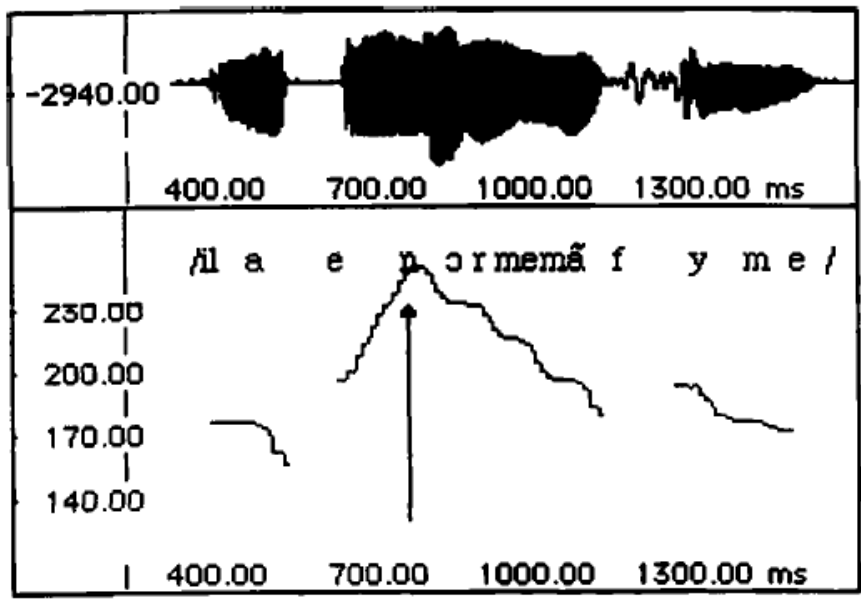

Abb. 6: Der Pfeil markiert die Fo-Gipfelposition auf der ersten, betonten Silbe des Adverbs.

Ähnliche Beobachtungen ergeben sich in den Beispielen (24a) und (25a). Im normalfokussierten Satz (24a) liegt der Fo-Gipfel zu Beginn der betonten Silbe am rechten Phrasenrand gefolgt von einem Grundfrequenzfall, wie in der folgenden Skizze dargestellt.

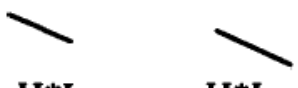

$H^{*} \mathrm{~L} \quad \mathrm{H}^{*} \mathrm{~L}$

Il a

fumé

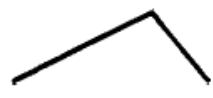

L $\quad \mathrm{H}^{*} \mathrm{~L}$

énormément 
Am rechten Satzrand fallen somit drei funktional-prosodische Eigenschaften zusammen: der Phrasenakzent des Adverbs, der Satzakzent und der für Deklarative typische finale tiefe Grenzton.

Eine weitere Beobachtung konnte bezüglich der Liaison getätigt werden. Bei Normalfokussierung trat keine Glottisverschlußinsertion vor dem Vokal der ersten Silbe des Adverbs auf.

Entsprechend den Voraussagen für das akzentuelle Verhalten unter den Bedingungen des Kontrastfokus verändert sich das Bild für (25a) dahingehend, daB der Phrasen- und somit Kontrastfokusakzent auf die erste Silbe des Adverbs fältt und auch durch eine angemessene Grundfrequenzbewegung angezeigt wird.

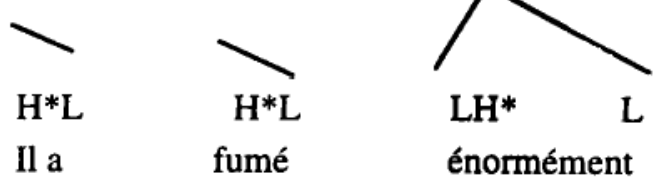

Bezüglich der GlottisverschluBinsertion konnte festgestellt werden, daB sie in $60 \%$ der aufgezeichneten Fälle auch tatsächlich eintrat. ${ }^{3}$

Untersuchungen zum Verhalten kontrastfokussierter Adverbien bezüglich der verketteten Liaison am rechten Phrasenrand stehen noch aus. ${ }^{4}$

\subsection{Auswertung und Zusammenfassung}

Das prosodische Verhalten des Französischen weicht vom Deutschen dahingehend ab, daß das Französische eine Sprache mit festen, vorhersagbaren Akzentpositionen ist. Der Akzent tritt immer an der Peripherie von Wörtern oder Phrasen auf - sowohl bei Normal- als auch bei Kontrastfokussierung.

Bei Normalfokussierung befindet sich der Akzent an der rechten Peripherie von Phrasen, bei Kontrastfokussierung wird der Phrasenakzent an die linke Grenze von Phrasen verschoben. In beiden Fällen wird dadurch eine Veränderung der prosodischen Phrasierung signalisiert. Bei

3 Das Korpus besteht aus Aufnahmen von 4 französischsprachigen Sprecherinnen und enthält u.a. weitere Adverbien mit initialem Vokal wie immodérément ${ }_{n}$ unmäBig”. Der Glottisverschluß 8 wurde phonetisch auf verschiedene Weise realisiert, z.B. durch Laryngalisierung.

4 Das sind z.B. Sātze wie: Is se sont trop aimés. (Sie haben sich zu sehr gemocht). 
Kontrastfokussierung wird eine prosodische Phrasierung erzeugt, die sich stark an der ursprünglichen syntaktischen Phrasierung orientiert. Die kontrastfokussierten Konstituenten konservieren damit ihre Autonomie, die Akzentverschiebung an den linken Phrasenrand bricht die normale Phrasierung auf und erlaubt zugleich eine Disambiguierung hinsichtlich der Informationsstruktur.

Der Zusammenhang zwischen Akzentplazierung und der damit einhergehenden Fokussierung sowie segmentalen phonologischen Regularitäten wie Liaison wird besonders bei initialer Betonung deutlich. Durch die Glottisverschlußeinfügung in den Onset einer vokalisch anlautenden betonten Silbe eines Adverbs wird diese Silbenposition gefüllt und eine Liaison unterbleibt.

Ist diese Silbe nicht betont, besteht auch keine Notwendigkeit, die Autonomie dieser Phrasengrenze aufrecht zu erhalten, da die prosodische Phrasierung in Sätzen mit konsequenter rechtsseitiger Phrasenbetonung normalfokussierten Sätzen entspricht.

Im nächsten Abschnitt möchte ich eine weitere Möglichkeit der akustischen Analyse unterschiedlich fokussierter Sätze andeuten. Ausgewählt wurde dazu ein kleines Korpus aus dem Russischen.

\section{Beobachtungen im Russischen}

Das Russische verfügt über äußerst variable Stellungseigenschaften für Akzente. Damit unterscheidet es sich vom Deutschen und vom Französischen, wobei das Russische einen Gegenpol zum Französischen bildet und das Deutsche zwischen diesen beiden Extremen liegt.

Die Schwierigkeiten, die mit der Erkennung von Phrasengrenzen anhand des Grundfrequenzverlaufes im Deutschen verbunden sind, können bei der Analyse des Russischen von vornherein durch ein anderes Vorgehen umgangen werden.

Das Ziel der Untersuchung besteht weiterhin darin, im akustischen Signal Indikatoren für die durch Fokussierung bedingte prosodische Phrasierung zu ermitteln. Wenn die Phrasierung nicht durch den Platz der Akzente angezeigt werden kann, bietet es sich an, nach Pausensignalen zu suchen und anhand derer Rückschlüsse auf die prosodische Phrasierung zu ziehen.

Die Verfahrensweise, die hier vorgestellt wird, orientiert sich an einer Beobachtung von Hayes/Lahiri (1991) im Bengali, wo durch bestimmte Assimilationsprozesse Phrasengrenzen determinierbar sind. Ähnliches ließ sich für bestimmte phonologische Prozesse im Russischen ermitteln. Dort 
kann innerhalb von phonologische Phrasen zwischen zwei Konsonanten Geminierung auftreten:

(28) $\mathrm{k}$ kasse

/k: asje/

zur Kasse

$$
\begin{aligned}
& \text { ob bereg } \\
& \text { /วb: jerEg/ } \\
& \text { über das Ufer }
\end{aligned}
$$

Dieser ProzeB tritt auch mit regressiver Stimmassimilation auf, d.h. der im Auslaut verhärtete ursprüngliche stimmhafte Konsonant wird unter der Bedingung der Geminierung wieder stimmhaft.

(30) iz zala

/iz: ala/

aus dem Saal

Diese Beobachtung wird hier als Grundlage für ein gegenüber den vorangehenden Untersuchungen modifiziertes Analyseverfahren genommen.

\subsubsection{Testaufbau}

Zunächst wurde davon ausgegangen, daB der syntaktische Kontext, d.h. die Wortfolge in den untersuchten Sätzen, stabil gehalten wird und die Konstituenten zweisilbige Wörter sind. ${ }^{5}$

(31) Subjekt + Verb + Objekt

Weiterhin wurde angestrebt, für jede Konstituente an den linken und rechten Worträndern das gleiche segmentale Material zu haben $(C=$ Konsonant, $\mathrm{V}=$ Vokal).

$$
\begin{aligned}
& \text { Subjekt }+ \text { Verb } \\
& C_{1} V_{n} V_{1}+C_{1} V_{n} V_{1}+C_{1}+C_{1} V_{n} V_{n} C_{1}
\end{aligned}
$$

Der Konsonant $\mathrm{C}_{\mathrm{n}}$ in der Wortmitte bleibt unspezifiziert, der Konsonant $\mathrm{C}_{1}$ dagegen wurde für diesen Test auf den stimmhaften Frikativ /z/ festgelegt. Daraus ergibt sich folgende Wortkombination:

$$
\begin{array}{lll}
\text { zavxoz } & \text { zavjoz } & \text { zakaz } \\
\text { /zavxoz/ } & \text { /zavjoz/ / zakaz/ } \\
\text { der Verwalter brachte } & \text { die Bestellung }
\end{array}
$$

Die Voraussage betrifft die prosodische Phrasierung unter dem Einfluß verschiedener Fokustypen. Bei Normalfokussierung werden alle Konstituenten des Satzes fokussiert, die prosodische Phrasierung sollte daher

5 Dieses Korpus wurde in Zusammenarbeit mit Uwe Junghanns (FAS) und Gerhild Zybatow (U. Leipzig) aufgebaut. 
den gesamten Satz oder wenigstens das Verb und das Objekt umfassen. Das Objekt sollte bei Kontrastierung eine eigene prosodische Phrase bilden und es sollte ein Grenzsignal zwischen Verb und Objekt erkennbar sein.

\subsubsection{Durchführung des Experiments}

Zur Festlegung der Informationsstruktur wurde wiederum die FrageAntwort-Methode verwendet. Als Antwort auf die Fragen diente der in seiner Wortstellung unveränderte Antwortsatz (33). Vorgestellt werden hier nur die Sequenzen bei Normalfokussierung (34) und Kontrastfokussierung (35).

$\mathrm{V}$ čjom delo?

Was ist passiert?

(34) Zavxoz zavjoz zakaz.

Zavxoz zavjoz zavtrak ili zakaz?

Brachte der Verwalter das Frühstück oder die Bestellung?

(35) Zavxoz zavjoz zakaz.

\subsubsection{Ermittlung der Daten}

Beide Sätze wurden aufgezeichnet und anhand des Oszillogramms analysiert. Ich gehe davon aus, daB sich die Geminierung stimmhafter Frikative im oszillographischen Bereich, d.h. anhand der Schalldruck-ZeitFunktion periodischer und aperiodischer Schwingungen ablesen läBt. Dieses Verfahren ist relativ einfach, wenn man berücksichtigt, daß stimmhafte Sequenzen einen periodischen Verlauf aufweisen, stimmlose Sequenzen dagegen keine periodischen Schwingungen haben. Das beruht auf der Tatsache, daß bei stimmlosen Lauten die Glottis nicht schwingt.

\subsubsection{Auswertung}

Das folgende Oszillogramm zeigt genau denjenigen Ausschnitt aus (34), der $\mathbf{z w i s c h e n ~ d e m ~ V e r b ~ u n d ~ d e m ~ O b j e k t ~ l i e g t . ~ E s ~ i s t ~ d e u t l i c h ~ z u ~ e r k e n n e n , ~}$ $\mathrm{da} \beta$ die letzten periodischen Schwingungen des Konsonanten $/ \mathrm{z} / \mathrm{des}$ Verbs ohne Unterbrechung in die ersten periodischen Schwingungen des ersten Konsonanten des Objektes überführt werden. 


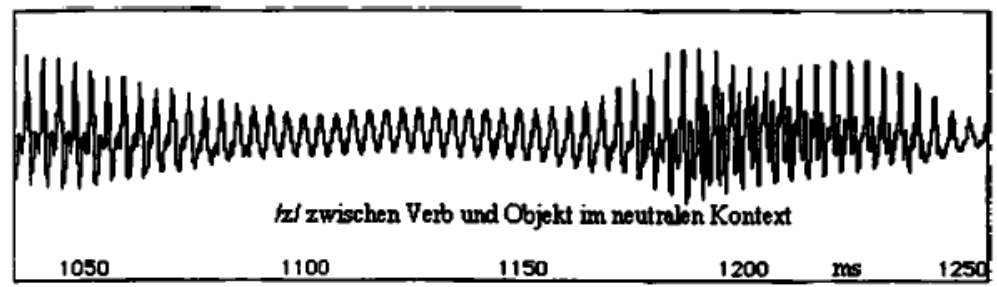

Abb. 7: Der nicht unterbrochene periodische Übergang von Verb zu Objekt für (34).

Völlig anders verhält sich das akustische Signal im gleichen Abschnitt zwischen dem Verb und dem Objekt in der Antwortsequenz (35). Hier ist deutlich zu erkennen, daß das periodische Signal durch eine aperiodische Sequenz unterbrochen wird.

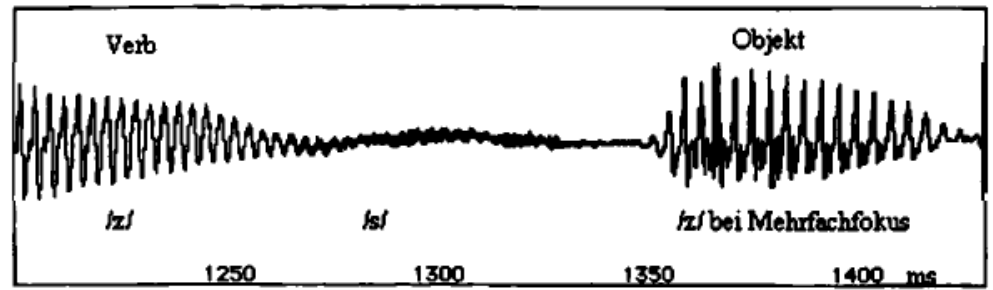

Abb. 8: Der entsonorisierte Übergang von Verb zu Objekt für (35).

Diese relativ lange aperiodische Sequenz, die in ihren spektralen Eigenschaften dem eines stimmlosen /s/ entspricht ${ }^{6}$, kann in diesem Fall als Grenzsignal interpretiert werden.

Obwohl die beiden untersuchten Sätze die gleiche syntaktische Strukturierung und die gleiche segmentale Umgebung an der Peripherie ihrer Konstituenten aufweisen, sind Unterschiede zwischen den verschiedenen Fokustypen und damit verbunden zwischen den daraus induzierten unterschiedlichen prosodischen Phrasierungseigenschaften erkennbar. Die o.g. Voraussage, daß bei Kontrastierung des Objektes dieses eine eigene prosodische Phrase bildet, konnte verifiziert werden.

6 Das Spektrogramm des relevanten Signalabschnittes wird hier nicht gezeigt. 
Mithin ergibt sich ein dem Französischen ähnliches Bild: Die Insertion eines Grenzsignals bedeutet die Aufspaltung der unter Normalfokussierung bestehenden $\mathrm{Phrasenverhältnisse.}{ }^{7}$ Ebenso wurde gezeigt, daß bei Normalfokussierung gröBere prosodische Einheiten gebildet werden, was darauf beruht, daß der fokussierte Bereich maximiert wird. Daraus folgt, daß zwischen den betroffenen Konstituenten keine solche Grenze signalisiert wird.

\section{$3.2 \quad$ Fazit}

Damit ergibt sich ein ähnliches Bild wie im Deutschen und Französischen. Werden innerhalb eines kontinuierlichen Signalverlaufs Grenzsignale eingefügt, verändert sich die prosodische Phrasierung, bedingt durch die Ẩnderung der Zuweisungsdomänen für Merkmale der verschiedenen Fokustypen. Wird eng oder kontrastfokussiert, sind hierarchisch sehr hoch angesiedelte Domänen nicht mehr erkennbar und auch nicht mehr notwendig. Wird bei Normalfokussierung dagegen die vom Fokusmerkmal dominierte Domäne optimiert, sind die darunterliegenden prosodischen Domänen nicht mehr erkennbar.

An drei Sprachen, die sich syntaktisch, informationsstrukturell und phonologisch unterschiedlich verhalten, wurde gezeigt, daß es markante $\mathrm{Zu}$ sammenhänge zwischen Akzentuierung und prosodischer Phrasierung geben kann. Das hängt damit zusammen, daß bereits in der Syntax operierende Fokusmerkmale auf der Phonologischen Ebene Einfluß auf die Positionierung und den tonalen Verlauf der Akzente nehmen können. Hinzukommt, daß je nach Fokusart der Einflußbereich dieses Merkmals unterschiedlich definiert ist.

Die Strategien zur Detektion von Phrasierungseinheiten im akustischen Signal müssen daher auf unterschiedliche Bedingungen im syntaktischen, informationsstrukturellen und phonologischen Bereich ausgerichtet werden. Es wurde gezeigt, daB anhand des Parameters der Grundfrequenz prosodische Phrasierung in Sätzen nur bedingt nachweisbar ist: im Deutschen im Fall der multiplen Fokussierung adjazent zueinander stehender Konstituenten, im Französischen eindeutig an der Grundfrequenzbewegung über der betonten Silbe. Für das Russische wurde von vornherein aufgrund phonologischer Vorüberlegungen ein anderer Weg gewählt. Es

7 Āhnlich dem Französischen würde eine Phrasengrenze zwischen Verb und Objekt auch eine solche zwischen Subjekt und Verb verlangen. Dies trifft für den hier vorgestellten Typ der Kontrastierung auch zu. Weitere Untersuchungen dazu für die multiple Fokussierung sind in Vorbereitung. 
konnte belegt werden, daB im Russischen an Phrasengrenzen Assimilationserscheinungen auftreten.

Den Ausgangspunkt für die akustische Erkennung von Phrasierungseinheiten bildete die Beobachtung, daB das Französische über eine festgelegte Position des lexikalischen Akzentes an der rechten Wortperipherie verfügt und bei Kontrastfokussierung von Adverbien der Akzent an die linke Peripherie verlagert wird. Wird der Akzent durch eine deutliche Grundfrequenzveränderung angezeigt, ist damit die Phrasierung mittels dieses Parameters auch möglich.

Deutsch und Russisch dagegen verfügen über andere Eigenschaften bezüglich der Position lexikalischer Akzente. Sie fallen nicht immer an die Wortperipherie, was induziert, daB nur in bestimmten Fällen die prosodische Phrasierung über den Fo-Parameter realisiert wird. Im Russischen zeigen Assimilationen Phrasierungen an; bezüglich des Deutschen muß auf die derzeit noch existierende Lücke in der Untersuchung von informationsstrukturell gesteuerten Assimilationsregeln und Glottisverschlußeinfügungen auf phrasaler Ebene verwiesen werden.

\section{Literatur}

Abraham, W. (1994): The Focus Null Hypothesis and the head-direction parameter: word compounding, numerals, and proper names. In: de Boer, A./de Hoop, H./de Swart, H. (eds.): Language and Cognition 2. S. 1-15.

Alter, K. (1994): Die französischen Interrogative und Exklamative - zum modularen Zusammenhang von Syntax und Phonologie. Diss. Uni. Leipzig.

Batliner, A. (1994): Prosody, focus, and focal structure: some remarks on methodology. In: Bosch, P./van der Sandt, R. (eds.): Focus/Natural language Processing. Vol. 1. Intonation and Syntax, Working Paper 6. Dec. 1994. Heidelberg. S. 11-28.

Caelen-Haumont, G. (1993): Stratégies des locuteurs en réponse à des consignes de lecture d'un texte: Analyse des interactions entre modèles syntaxiques, sémantiques, pragmatiques et paramètres prosodiques. Grenoble.

Cinque, G. (1993): A null theory of phrase and compound stress. In: Linguistic Inquiry 24, S. 239-398.

Cruttenden, A. (1986): Intonation. Cambridge textbooks in linguistics. Cambridge.

Dogil, G. (1995): Word prosodic systems in Slavic Languages. In: v.d. Hulst, H. (ed.) (to app.): Chapt. $11 \& 12$.

Féry, C. (1993): German Intonational Patterns. Tübingen. (Linguistische Arbeiten 285).

Grice, M. (1995): The intonation of interrogation in Palermo Italian. Tübingen. (Linguistische Arbeiten 334). 
Gussenhoven, C. (1994): Focus and sentence accent in English. In: Bosch, P./van der Sandt, R. (eds.): Focus \& Natural language Processing. Vol. 1. Intonation and Syntax. Working Paper 6. Dec. 1994, Heidelberg. S. 83-92.

Hayes, B./Lahiri, A, (1991): Bengali Intonational Phonology. In: Natural Language and Linguistic Theory 9, S. 47-96.

Hirst, D. (1993): Detaching Intonational Phrases from Syntactic Structure. In: Linguistic Inquiry 23, S. 781-788.

Jacobs, J. (1994): Informational Autonomy. In: Bosch, P./van der Sandt, R. (eds.): Focus \& Natural language Processing. Vol. 1. Intonation and Syntax. Working Paper 6. Dec. 1994. Heidelberg. S. 113-132.

Lyche, C./Girard, F. (1995): Le mot retrouvé. In: Durand, J./Hintze M.-A. (eds.): French Phonology: Morae, syllables, words. S. 205-221. (Lingua, vol. 95).

Möbius, B. (1993): Ein quantitatives Modell der deutschen Intonation: Analyse und Synthese von Grundfrequenzverläufen. Tübingen. (Linguistische Arbeiten 305).

Nespor, M./Vogel, I. (1986): Prosodic Phonology. Dordrecht.

Pierrehumbert, J. (1980): The Phonetics and Phonology of English Intonation. Doctoral diss. MIT.

Rooth, M. (1992): A theory of focus interpretation. In: Natural Language Semantics 1, S. 75-116.

Tillmann, H.G. (mit Mansell, P.) (1980): Phonetik. Lautsprachliche Zeichen, Sprachsignale und lautsprachlicher Kommunikationsprozeß. Stuttgart.

Tranel, B. (1987): The sounds of French. Cambridge.

Tranel, B. (1995): French final consonants and non-linear phonology. In: Durand, J./Hintze, M.-A. (eds.): French Phonology: Morae, syllables, words. S. 131-168. (Lingua, vol. 95).

Uhmann, S. (1987): Fokussierung und Intonation. Diss. Uni. Konstanz. 